PROCEEDINGS OF THE

AMERICAN MATHEMATICAL SOCIETY

Volume 138, Number 1, January 2010, Pages 253-261

S 0002-9939(09)10036-9

Article electronically published on August 19, 2009

\title{
ON THE LOCAL ANALYTIC INTEGRABILITY AT THE SINGULAR POINT OF A CLASS OF LIÉNARD ANALYTIC DIFFERENTIAL SYSTEMS
}

\author{
JAUME LLIBRE AND CLÀUDIA VALLS
}

(Communicated by Yingfei Yi)

\begin{abstract}
We consider the Liénard analytic differential systems $\dot{x}=y, \dot{y}=$ $-c x-f(x) y$, with $c \in \mathbb{R}$ and $f: \mathbb{R} \rightarrow \mathbb{R}$ an analytic function. Then for such systems we characterize the existence of local analytic first integrals in a neighborhood of the singular point located at the origin.
\end{abstract}

\section{Introduction AND STATEMENT OF THE MAIN RESUlts}

One of the more classical problems in the qualitative theory of planar analytic differential systems in $\mathbb{R}^{2}$ is to characterize the existence of analytic first integrals in a neighborhood of an isolated singular point.

One of the best and oldest results in this direction is the analytic nondegenerate center theorem. In order to be more precise we recall some definitions. A singular point is a nondegenerate center if it is a center with eigenvalues purely imaginary. If a real planar analytic system has a nondegenerate center, then after an affine change of variables and a rescaling of the independent variable $t$, it can be written in the form

$$
\begin{aligned}
& \dot{x}=y+X(x, y), \\
& \dot{y}=-x+Y(x, y),
\end{aligned}
$$

where $X(x, y)$ and $Y(x, y)$ are real analytic functions without constant and linear terms defined in a neighborhood of the origin. The dot will always denote derivative with respect to the independent variable $t$.

Let $U$ be an open subset of $\mathbb{R}^{2}, H: U \rightarrow \mathbb{R}$ be a nonconstant analytic function and $\mathcal{X}$ be an analytic vector field defined on $U$. Then $H$ is an analytic first integral of $\mathcal{X}$ in $U$ if $H$ is constant on the solutions of $\mathcal{X}$, i.e. if $\left.\mathcal{X} H\right|_{U}=0$. If $U=\mathbb{R}^{2}$, then $H$ is called a global analytic first integral of $\mathcal{X}$.

The next result is due to Poincaré [7, [8] and Liapunov [5]; see also Moussu [6].

Theorem 1 (Analytic nondegenerate center theorem). The analytic differential system (11) has a nondegenerate center at the origin if and only if there exists an analytic first integral defined in a neighborhood of the origin.

Received by the editors February 21, 2009, and, in revised form, April 30, 2009.

2000 Mathematics Subject Classification. Primary 34C05, 34A34, 34C14.

Key words and phrases. Analytic integrability, local analytic integrability, Liénard differential system. 
One of the more studied differential equations is the so-called generalized Liénard equation

$$
\ddot{x}+f(x) \dot{x}+g(x)=0 .
$$

Such dynamical systems appear very often in several branches of the sciences, such as biology, chemistry, mechanics, electronics, etc. Here we will restrict our studies to the case $g(x)=c x$.

The differential equation (2) of second order with $g(x)=c x$ can be written as the equivalent 2-dimensional Liénard differential system of first order

$$
\dot{x}=y, \quad \dot{y}=-c x-f(x) y .
$$

For $c=1$ the Liénard differential systems (3) are called the classical Liénard systems. The main objective of this paper is to study the analytical integrability of the Liénard systems (3) depending on the analytic function $f(x)$ and on $c \in \mathbb{R}$. We assume that $f(x) \not \equiv 0$; otherwise system (3) becomes a differential linear system. If $c=0$, then the Liénard differential system (3) becomes a differential equation in separable variables and its integration is easy, so we do not consider this case here. Moreover we will see in Section 2 that after a rescaling of the variables $x, y, t$ we always go over the cases $c= \pm 1$. In the rest of the paper we consider only these two possibilities.

As is well known, the function

$$
F(x)=\int_{0}^{x} f(s) d s
$$

will be useful in the study of the Liénard differential systems (3).

The main result of this paper is the following.

Theorem 2. The Liénard differential system (3) with $c= \pm 1$ has a local analytic first integral in a neighborhood of the origin if and only if one of the following conditions holds:

(a) $c=1, f(0)=0$ and $F(x)$ is an even function of $x$;

(b) $c=-1, f(0)=0$ and $F(x)$ is an even function of $x$;

(c) $c=-1, f(0)=(p-q) / \sqrt{p q}$ for some positive coprime integers $p$, $q$ with $p>q$, and $f(x)=f(0)$ for all $x \in \mathbb{R}$ (in this case system (3) has the polynomial first integral $\left.H=(\sqrt{p q} y-q x)^{q}(\sqrt{p q} y+p x)^{p}\right)$.

The paper has been structured as follows: Sections 2, 3 and 4 are dedicated to preliminary results. Using all of them in Section [5 we prove Theorem 2 .

\section{Preliminary Results}

Under the assumption that $c \neq 0$ we shall show that we can reduce the study of the Liénard differential systems (3) to the cases $c= \pm 1$.

Lemma 3. A Liénard differential system (3) with $c \neq 0$ after a rescaling of the variables $(x, y, t)$ becomes another Liénard differential system (3) with $c= \pm 1$.

Proof. Assume that $c>0$. Then we consider the rescaling $(X, Y, T)=(\sqrt{c} x, y$, $\sqrt{c} t$ ). System (3) in the new variables is written again as a system (3) with $c=1$.

Assume that $c<0$. Then doing the rescaling $(X, Y, T)=(\sqrt{-c} x, y, \sqrt{-c} t)$, system (3) in the new variables becomes again another system (3) with $c=-1$. 
In the rest of this section we shall introduce three results that will be used throughout the paper. The first two results are also true for complex analytic vector fields, but since here we only work with real analytic vector fields, we state them only for this last class of vector fields.

The following result is due to Poincaré and its proof can be found in [8]; see also [3].

Theorem 4. Let $\mathcal{X}=\mathcal{X}(x, y)$ be a real analytic vector field defined in a neighborhood of the origin such that $\mathcal{X}(0,0)=0$. Assume that the eigenvalues $\lambda_{1} \neq 0$ and $\lambda_{2} \neq 0$ at the origin of $\mathcal{X}$ do not satisfy any resonance condition of the form

$$
\lambda_{1} k_{1}+\lambda_{2} k_{2}=0 \quad \text { for } \quad k_{1}, k_{2} \in \mathbb{Z}^{+} \quad \text { with } \quad k_{1}+k_{2}>0 .
$$

Then $\mathcal{X}$ has no local analytic first integrals in a neighborhood of the origin.

We must mention that the singular points appearing in the statements of Theorem 4 can be real or complex, independently of the fact that we are working with real vector fields.

We have the following nice characterization of the centers at the origin for the Liénard systems (3) with $c=1$; see for instance [1].

Theorem 5 (Center Theorem for analytic Liénard systems). Let $f(x)$ be an analytic function defined in a neighborhood of zero. Then the Liénard differential system (3) with $c=1$ has a nondegenerate center at the origin if and only if $f(0)=0$ and $F(x)$ is an even function of $x$.

\section{CASE $f(0) \neq 0$}

In this section we consider system (3) with $f(0) \neq 0$. We studied the analytic integrability of it in the next four propositions.

As is usual, $\mathbb{Z}^{+}$will denote the set of nonnegative integers, $\mathbb{Z}^{-}$will denote the set of negative integers, $\mathbb{Q}^{+}$will denote the set of nonnegative rationals, and $\mathbb{Q}^{-}$ will denote the set of negative rationals.

Proposition 6. Let $f(x)$ be an analytic function defined in a neighborhood of the origin such that $f(0) \neq 0$ and $c / f(0)^{2} \notin \mathbb{Q}^{-}$. Then system (3) with $c= \pm 1$ has no local analytic first integrals in a neighborhood of the origin.

Proof. We note that system (3) has the singular point $(0,0)$. If $\mathcal{X}=(y,-c x+f(x) y)$, then the eigenvalues of $D \mathcal{X}(0,0)$ are

$$
\lambda_{1}=\frac{f(0)+\sqrt{f(0)^{2}-4 c}}{2} \text { and } \quad \lambda_{2}=\frac{f(0)-\sqrt{f(0)^{2}-4 c}}{2} .
$$

Clearly

$$
\lambda_{1}+\lambda_{2}=f(0) \text { and } \lambda_{1} \lambda_{2}=c .
$$

Suppose that there exist positive integers $k_{1}$ and $k_{2}$ such that $k_{1} \lambda_{1}+k_{2} \lambda_{2}=0$. Then $\lambda_{1}=-\alpha \lambda_{2}$ with $\alpha$ a positive rational. The two equalities of (4) become

$$
f(0)=(1-\alpha) \lambda_{2} \text { and } \quad c=-\alpha \lambda_{2}^{2} .
$$

We have

$$
\frac{f(0)^{2}}{c}=-\frac{(1-\alpha)^{2}}{\alpha} \in \mathbb{Q}^{-} .
$$


Note that $\alpha \neq 1$ because $f(0) \neq 0$, and $\alpha \neq 0$ because $c \neq 0$. Therefore, since by assumptions $f(0)^{2} / c \notin \mathbb{Q}^{-}$, we cannot have $k_{1} \lambda_{1}+k_{2} \lambda_{2}=0$. Then the proposition follows using Theorem 4 .

Proposition 7. Let $f(x)$ be an analytic function defined in a neighborhood of the origin such that $f(0) \neq 0$ and $c / f(0)^{2}=-\alpha \in \mathbb{Q}^{-}$with $\alpha \neq p q /(p-q)^{2}$ for some coprimes $p, q \in \mathbb{Z}^{+}$and $p>q$. Then system (3) with $c=-1$ has no local analytic first integrals in a neighborhood of the origin.

Proof. Doing the rescaling $(X, Y, T)=(f(0) x, y, f(0) t)$, system (3) becomes

$$
\dot{x}=y, \quad \dot{y}=\alpha x-y+\text { h.o.t. }
$$

where we have written again $(x, y, t)$ instead of $(X, Y, T)$, and where h.o.t. means terms of higher order. We assume that $H=H(x, y)$ is a local analytic first integral in a neighborhood of the origin. We write it as $H=\sum_{k \geq 1} H_{k}(x, y)$, where $H_{k}$ are homogeneous polynomials of degree $k$. We will show by induction that

$$
H_{k}=0 \quad \text { for } k \geq 1 \text {. }
$$

Then clearly from (5) we will obtain that system (3) has no local analytic first integrals, and the proof of the proposition will be done.

Since $H$ is a first integral, it must satisfy

$$
(\alpha x-y+\text { h.o.t. }) \frac{\partial H}{\partial y}+y \frac{\partial H}{\partial x}=0 .
$$

Now we will prove (5) using induction with respect to $k$. The terms of degree one in (6) must satisfy

$$
(\alpha x-y) \frac{\partial H_{1}}{\partial y}+y \frac{\partial H_{1}}{\partial x}=0 .
$$

Clearly, since $\alpha \neq 0$ we have that $\partial H_{1} / \partial y=0$. Then we also obtain $\partial H_{1} / \partial x=0$ and thus $H_{1}=0$, which proves (5) for $k=1$. Now we assume that (5) is true for $k=1, \ldots, j-1$ with $j \geq 2$ and we will prove it for $k=j$. By the induction hypothesis, the terms of order $j$ in (6) must satisfy

$$
(\alpha x-y) \frac{\partial H_{j}}{\partial y}+y \frac{\partial H_{j}}{\partial x}=0 .
$$

Therefore, either $H_{j}=0$ or $H_{j}$ is a first integral of the linear differential system

$$
\dot{x}=y, \quad \dot{y}=\alpha x-y .
$$

Computing a first integral of this system we obtain that it must be a function $h(G)$ of $G$ with

$$
G=\left(\frac{1}{2}(1-\sqrt{1+4 \alpha}) x+y\right)^{-1+\sqrt{1+4 \alpha}}\left(\frac{1}{2}(1+\sqrt{1+4 \alpha}) x+y\right)^{1+\sqrt{1+4 \alpha}} .
$$

The unique possibility in order that $h(G)$ be a polynomial is that

$$
-1+\sqrt{1+4 \alpha}=\frac{n_{1}}{n_{2}} \text { and } 1+\sqrt{1+4 \alpha}=\frac{n_{3}}{n_{2}}, n_{1}, n_{2}, n_{3} \in \mathbb{Z}^{+}, n_{3} \neq n_{1} .
$$

Then we have that

$$
\frac{n_{1}}{n_{2}}+1=\frac{n_{3}}{n_{2}}-1, \quad \text { that is, } \quad n_{2}=\frac{n_{3}-n_{1}}{2} .
$$


Hence

which yields

$$
\sqrt{1+4 \alpha}=\frac{n_{1}+n_{3}}{n_{3}-n_{1}}
$$

$$
\alpha=\frac{1}{4}\left(\left(\frac{n_{1}+n_{3}}{n_{3}-n_{1}}\right)^{2}-1\right)=\frac{n_{1} n_{3}}{\left(n_{3}-n_{1}\right)^{2}}, \quad n_{1}, n_{3} \in \mathbb{Z}^{+}, n_{1} \neq n_{3},
$$

in contradiction with the assumptions on $\alpha$. Note that we can assume $n_{3}>n_{1}$. Furthermore, $n_{1}$ and $n_{3}$ are coprime, since otherwise, setting $n_{1}=$ g.c.d. $\left\{n_{1}, n_{3}\right\} \hat{n}_{1}$ and $n_{3}=$ g.c.d. $\left\{n_{1}, n_{3}\right\} \hat{n}_{3}$, we get

$$
\alpha=\frac{\text { g.c.d. }\left\{n_{1}, n_{3}\right\}^{2} \hat{n}_{1} \hat{n}_{3}}{\text { (g.c.d. } \left.\left\{n_{1}, n_{3}\right\} \hat{n}_{1}+\text { g.c.d. }\left\{n_{1}, n_{3}\right\} \hat{n}_{3}\right)^{2}}=\frac{\hat{n}_{1} \hat{n}_{3}}{\left(\hat{n}_{1}-\hat{n}_{3}\right)^{2}} .
$$

Since all first integrals are functions of $G$ it is clear that $H_{j}$ cannot be a homogeneous polynomial of degree $j$. Hence $H_{j}=0$ and the induction process has ended.

Proposition 8. Let $f(x)=f(0) \neq 0$ for all $x \in \mathbb{R}$ with $1 / f(0)^{2}=p q /(p-q)^{2}$ for some coprime $p, q \in \mathbb{Z}^{+}$and $p>q$. Then system (3) with $c=-1$ has the global analytic first integral

$$
H=(\sqrt{p q} y-q x)^{q}(\sqrt{p q} y+p x)^{p} .
$$

Proof. Under the assumptions of the proposition, system (3) becomes

$$
\dot{x}=y, \quad \dot{y}=x-\frac{p-q}{\sqrt{p q}} y .
$$

Now the proposition follows by direct computations.

Proposition 9. Let $f(x) \neq f(0) \neq 0$ for some $x \in \mathbb{R}$ with $1 / f(0)^{2}=p q /(p-q)^{2}$ for some coprimes $p, q \in \mathbb{Z}^{+}$and $p>q$. Then system (3) with $c=-1$ has no local analytic first integrals in a neighborhood of the origin.

Proof. Under the assumptions of the proposition, system (3) becomes

$$
\dot{x}=y, \quad \dot{y}=x-\frac{p-q}{\sqrt{p q}} y-\sum_{j \geq 1} \frac{f^{(j)}(0)}{j !} x^{j} y .
$$

Making the change of variables

$$
Y=y+\frac{p-q}{\sqrt{p q}} x+\sum_{j \geq 1} \frac{f^{(j)}(0)}{(j+1) !} x^{j+1}, \quad X=x,
$$

system (8) becomes

$$
\dot{X}=Y-\frac{p-q}{\sqrt{p q}} X-\sum_{j \geq 1} \frac{f^{(j)}(0)}{(j+1) !} X^{j+1}, \quad \dot{Y}=X .
$$

Renaming $y=X$ and $x=Y$, system (9) becomes

$$
\dot{x}=y, \quad \dot{y}=x-\frac{p-q}{\sqrt{p q}} y-\sum_{j \geq 1} \frac{f^{(j)}(0)}{(j+1) !} y^{j+1} .
$$

Now let

$$
u=\sqrt{p q} y-q x, v=\sqrt{p q} y+p x, \quad \text { i.e. } \quad x=\frac{v-u}{p+q}, y=\frac{p u+q v}{\sqrt{p q}(p+q)} .
$$


Then system (10) becomes

$$
\begin{aligned}
& \dot{u}=-\frac{p}{\sqrt{p q}} u-\sqrt{p q} \sum_{j \geq 1} \frac{f^{(j)}(0)}{(j+1) !}\left(\frac{p u+q v}{\sqrt{p q}(p+q)}\right)^{j+1}, \\
& \dot{v}=\frac{q}{\sqrt{p q}} v-\sqrt{p q} \sum_{j \geq 1} \frac{f^{(j)}(0)}{(j+1) !}\left(\frac{p u+q v}{\sqrt{p q}(p+q)}\right)^{j+1} .
\end{aligned}
$$

We change from the variables $(u, v)$ to the new variables $(u, T)$, where

$$
T=u^{q} v^{p}, \quad \text { that is, } \quad v=T^{1 / p} u^{-q / p} .
$$

Then we have from (11) that

$$
\begin{aligned}
& \dot{u}=-\frac{p}{\sqrt{p q}} u-\sqrt{p q} \sum_{j \geq 1} \frac{f^{(j)}(0)}{(j+1) !}\left(\frac{p u+q T^{1 / p} u^{-q / p}}{\sqrt{p q}(p+q)}\right)^{j+1}, \\
& \dot{T}=u^{(q-p) / p} T^{(p-1) / p} \sum_{j \geq 1} \frac{f^{(j)}(0)}{(j+1) !}\left(\frac{p u+q T^{1 / p} u^{-q / p}}{\sqrt{p q}(p+q)}\right)^{j+2} .
\end{aligned}
$$

Let $H=H(x, y)$ be a formal first integral of system (8). Then $\hat{H}(u, v)=H(x, y)$ is a formal first integral of system (11) and $\tilde{H}(u, T)=\hat{H}(u, v)$ is a first integral of system (12). Writing $\hat{H}(u, v)=\sum_{j \geq 0} H_{j}(u) v^{j}$ with $H_{j}$ a formal series in $v$, we can write $\tilde{H}(u, T)$ as

$$
\tilde{H}=\tilde{H}(u, T)=\sum_{j \geq 0} \tilde{H}_{j}(u) T^{j / p},
$$

where $\tilde{H}_{j}(u)=H_{j}(u) u^{-j q / p}$. Since $\tilde{H}$ is a first integral we can assume that it has no constant term. Note that $\tilde{H}$ satisfies

$$
\dot{T} \frac{\partial \tilde{H}}{\partial T}+\dot{u} \frac{\partial \tilde{H}}{\partial u}=0
$$

with $(\dot{T}, \dot{u})$ as in (12). We will show by induction that

$$
\tilde{H}_{j}(u)=0 \text { for } j \geq 0 \text {. }
$$

Note that to conclude the proof of the proposition it is enough to show that (14) holds.

First we note that equation (13) restricted to $T=0$ becomes

$$
\frac{1}{p} u^{(q-p) / p} \sum_{j \geq 0} \frac{f^{(j)}(0)}{(j+1) !}\left(\frac{p u}{\sqrt{p q}(p+q)}\right)^{j+2} \tilde{H}_{0}^{\prime}(u)=0,
$$

where the prime indicates derivative with respect to $u$. From the expression above we get that $\tilde{H}_{0}$ is a constant, and since $\tilde{H}$ has no constant term, we must have $\tilde{H}_{0}=0$. This proves (14) for $j=0$.

Now we assume that (14) is satisfied for $j=0, \ldots, n-1$ with $n \geq 1$, and we shall prove it for $j=n$. By the induction hypothesis we have

$$
\tilde{H}=\sum_{j \geq 0} \tilde{H}_{j+n}(u) T^{(j+n) / p} .
$$


Now equation (13) becomes

$$
\begin{aligned}
& u^{(q-p) / p} T^{(p-1) / p}\left(\sum_{j \geq 1} \frac{f^{(j)}(0)}{(j+1) !}\left(\frac{p u+q T^{1 / p} u^{-q / p}}{\sqrt{p q}(p+q)}\right)^{j+2}\right) \\
& \cdot\left(\sum_{j \geq 0} \tilde{H}_{j+n}(u) \frac{j+n}{p} T^{(j+n-p) / p}\right) \\
& -\left(\frac{p}{\sqrt{p q}} u+\sqrt{p q} \sum_{j \geq 1} \frac{f^{(j)}(0)}{(j+1) !}\left(\frac{p u+q T^{1 / p} u^{-q / p}}{\sqrt{p q}(p+q)}\right)^{j+1}\right) \\
& \cdot\left(\sum_{j \geq 0} \tilde{H}_{j+n}^{\prime}(u) T^{(n+j) / p}\right)=0,
\end{aligned}
$$

where the prime denotes derivative with respect to the variable $u$. After simplifying by $T^{(n-1) / p}$ equation (15), we restrict it to $T=0$ obtaining

$$
\frac{n}{p} u^{(q-p) / p}\left(\sum_{j \geq 1} \frac{f^{(j)}(0)}{(j+1) !}\left(\frac{p u}{\sqrt{p q}(p+q)}\right)^{j+2}\right) \tilde{H}_{n}(u)=0 .
$$

Therefore $\tilde{H}_{n}(u)=0$. This proves (14) for $j=n$. In short the proposition is proved.

$$
\text { 4. CASE } f(0)=0, c=-1
$$

In this section we prove the following proposition.

Proposition 10. Let $f(x)$ be an analytic function defined in a neighborhood of the origin such that $f(0)=0$. Then system (3) with $c=-1$ has a local analytic first integral in a neighborhood of the origin if and only if $F(x)$ is an even function of $x$.

Proof. Making the transformation $(X, Y)=(x, y+F(x))$, system (3) with $c=-1$ and $f(0)=0$ becomes

$$
\dot{x}=y-F(x), \quad \dot{y}=x, \quad \text { where } \quad F(x)=\sum_{j \geq 1} a_{j} x^{j}, \quad a_{j}=\frac{f^{(j-1)}(0)}{j !},
$$

where we have written $(x, y)$ instead of $(X, Y)$.

We first show that if $F$ is an even function of $x$, that is, $F(x)=\sum_{j>1} a_{2 j} x^{2 j}$, then system (16) has a local analytic first integral in a neighborhood of the origin. Indeed with the change of variables

$$
u=y, \quad v=-i x, \quad \tau=-i t,
$$

we have

$$
u^{\prime}=-v, \quad v^{\prime}=u-\sum_{j \geq 1} \tilde{a}_{2 j} v^{2 j}
$$

where ' denotes the derivative with respect to $\tau$ and $\tilde{a}_{2 j}=(-1)^{j} a_{2 j}$. Note that $u$ is real and that $v$ and $\tau$ are pure imaginary. Note that on the plane $\mathbb{R} \times i \mathbb{R}$ we have that system (18) is a center because it has a focus or a center at the origin and it is invariant under the symmetry $(u, v, \tau) \rightarrow(u,-v,-\tau)$. Therefore it has a local 
analytic first integral $G: \mathbb{R} \times i \mathbb{R}$ of the form $G=G(u, v)=G\left(u^{2}+v^{2}\right)$; see [4, 2], 9. Furthermore, since $\tilde{a}_{2 j} \in \mathbb{R}$ we get that $G\left(u^{2}+v^{2}\right)=\sum_{j \geq 1} b_{j}\left(u^{2}+v^{2}\right)^{j}$ with $b_{j} \in \mathbb{R}$. Note that $d G / d \tau=0$.

Since $v^{2}=-x^{2}$ (see (17)) we have that system (16) has a local analytic first integral in a neighborhood of the origin in the form $H=H(x, y)=G\left(y^{2}-x^{2}\right) \in \mathbb{R}$. Note that $d H / d t=(d G / d \tau)(d \tau / d t)=0$.

Now we shall prove the converse. Assume that system (3) with $c=-1$ has a local analytic first integral in a neighborhood of the origin. Consequently system (16) also has a local analytic first integral in a neighborhood of the origin. Therefore the system

$$
u^{\prime}=-v, \quad v^{\prime}=u-F(i v),
$$

obtained from system (16) after doing the change of variables (17), has a local analytic first integral in a neighborhood of the origin. Hence, by Theorem 5 it follows that $F(x)$ is an even function. So the proposition is proved.

\section{Proof of Theorem 2}

Finally we prove the main result of the paper.

Proof of Theorem 2(a). This follows directly from Theorems 5 and 1.

Proof of Theorem 2(b). This follows directly from Proposition 10.

Proof of Theorem 2(c). For $c=1$ and $f(0) \neq 0$ it follows from Proposition 6 that system (3) has no local analytic first integrals in a neighborhood of the origin.

For $c=-1$ and $f(0) \neq 0$ we get from Propositions 6 , 7, 8 and 9 that system (3) has a local first integral in a neighborhood of the origin if and only if $f(0)=$ $(p-q) / \sqrt{p q}$ for some positive coprime integers $p, q$ with $p>q$, and $f(x)=f(0)$ for all $x \in \mathbb{R}$.

\section{ACKNOWLEDGMENTS}

We thank the referee for an observation which allowed us to simplify a little the proof of statement (a) of Theorem 2 .

The first author has been supported by the grants MEC/FEDER MTM 200803437 and CIRIT 2009SGR 410. The second author has been supported by the Center for Mathematical Analysis, Geometry, and Dynamical Systems by the grant FCT-SFRH/BPD/26465/2006.

\section{REFERENCES}

1. C. Christopher, An algebraic approach to the classification of centers in polynomial Liénard systems, J. Math. Anal. Appl. 229 (1999), 319-329. MR.1664344 (99k:34056)

2. A. Cima, A. Gasull and F. Mañosas, Cyclicity of a family of vector fields, J. Math. Anal. Appl. 196 (1995), 921-937. MR1365231 (96j:58143)

3. S.D. FuRTA, On non-integrability of general systems of differential equations, Z. Angew Math. Phys. 47 (1996), 112-131. MR1408674 (97f:34003)

4. P. Joyal and C. Rousseau, Saddle quantities and applications, J. Diff. Equations 78 (1989), 374-399. MR992152 (90b:58225)

5. M.A. Liapunov, Problème général de la stabilité du mouvement, Ann. of Math. Stud., 17, Princeton University Press; Oxford University Press, London, 1947. MR0021186 (9:34j)

6. R. Moussu, Une démonstration géométrique d'un théorème de Lyapunov-Poincaré, Astérisque 98-99 (1982), 216-223. MR724449 (85g:58012) 
7. H. Poincaré, Mémoire sur les courbes définies par les équations différentielles, Journal de Mathématiques 37 (1881), 375-422; Oeuvres de Henri Poincaré, vol. I, Gauthier-Villars, Paris, 1951, pp. 3-84.

8. H. Poincaré, Sur l'intégration des équations différentielles du premier order et du premier degré $I$ and II, Rendiconti del circolo matematico di Palermo 5 (1891), 161-191; 11 (1897), $193-239$.

9. C. Zuppa, Order of cyclicity of the singular point of Liénard's polynomial vector fields, Bol. Soc. Brasil. Mat. 12 (1981), 105-111. MR688192 (84h:58126)

Departament de Matemàtiques, Universitat Autònoma de Barcelona, 08193 Bellaterra, Barcelona, Catalonia, Spain

E-mail address: jllibre@mat.uab.cat

Departamento de Matemática, Instituto Superior Técnico, 1049-001 Lisboa, PortuGAL

E-mail address: cvalls@math.ist.utl.pt 